\title{
Editorial for Special Issue on 3D Printing
}

\author{
Sergey Ishutov ${ }^{1} \cdot$ Franciszek Hasiuk $^{2}$
}

Published online: 21 June 2019

(c) Springer Nature B.V. 2019

Understanding geological processes at the pore scale has significant implications to natural phenomena related to flow in porous media (e.g., groundwater, hydrocarbons, and $\mathrm{CO}_{2}$ sequestration) and associated geomechanical properties. Despite advances in numerical and experimental methods for analyzing pore systems, correlation of petrophysical properties across multiple scales of investigation (e.g., between seismic data, core samples, thin-section images, and SEM images) is still challenging. Currently, we are in the initial stage of applying $3 \mathrm{D}$ printing technology in the geosciences to create physical models from digital designs of porous media that can be experimentally investigated and iteratively compared to numerical simulations. This special issue presents breakthroughs on reproduction of porous media using 3D printing. While many 3D printers manufacture models layer-by-layer, the physical and chemical properties of 3D printing materials, the process itself, and the post-processing methods employed vary widely.

Studies in the special issue are grouped into two sections according to the base material used in each 3D printing technique: (1) polymer-based 3D printing and (2) powder-based 3D printing. These studies provide the extent to which 3D printing techniques (binder jet, polyjet, and stereolithography) can manufacture useful models of porous media. Results presented herein emphasize validating the accuracy and resolution of each technique by testing the 3D printers with simple models (built from digital designs) and natural models (built from computed tomography data of natural porous rocks). With future advances in 3D printing resolution and materials, the fidelity to reproduce pore systems of natural rocks will improve. In addition, nomenclature on common 3D printing terms is provided for better comprehension of manufacturing techniques and processes.

$凶$ Sergey Ishutov

ishutov@ualberta.ca

http://www.rgrg.ca/

Franciszek Hasiuk

franek@kgs.ku.edu

1 Department of Civil and Environmental Engineering, Reservoir Geomechanics Research Group (RG)2, University of Alberta, 9211-116 Street NW, Edmonton, AB T6G 1H9, Canada

2 Kansas Geological Survey, University of Kansas, Lawrence, KS 66047, USA 


\section{Polymer-Based 3D Printing}

This section contains studies with models 3D-printed in polymeric materials for applications in flow experiments with porous media. Resins are used as the base material in photopolymer and stereolithographic 3D printing. The physical and chemical properties of resins can be modified to alter the surface properties of the resulting pore network, which is not feasible with natural rocks.

Ishutov presents an integrated analysis of uncured resins and cured models to evaluate the accuracy and resolution of the stereolithographic printing for porous models. The repeatability of the same pore network 3D-printed from tomographic data of Fontainebleau sandstone is tested in six resins of varying viscosity and resulting transport properties (e.g., porosity, pore sizes, and wettability).

Watson et al. compare a numerical simulation of a small-scale, single-phase flow with two micromodels 3D-printed with the same geometry in polymethyl and resin. The results demonstrate variability in print geometry and surface properties between the samples that need to be improved in order to achieve higher fidelity to numerical simulations.

Ahkami et al. perform numerical and experimental fluid flow using a fractured porous medium 3D-printed in plastic. The authors propose a new temporo-ensemble particle image velocimetry method to track fluid flow in fractures as well as interaction between fractures and matrices.

Suzuki et al. compare flow experiments on fractured porous media 3D-printed in polymer with numerical simulations using computational fluid dynamics based on the Navier-Stokes equations. The results show validation of an equivalent permeability model based on the cubic law with a tracer transport experiment.

\section{Powder-based 3D printing}

This section contains studies with models 3D-printed in powder materials for applications in flow, geomechanical, and coupled flow-deformation experiments. The powders used in $3 \mathrm{D}$ printing can have grain textures and surface chemistry and mineralogy similar to natural rocks. Therefore, this material may have the best capability to reproduce pore architecture analogous to that found in natural sedimentary rocks. While powders can be composed of minerals, metals, or polymers, their surface properties can be altered using chemical coatings to tune transport or geomechanical properties.

Hasiuk presents comprehensive analysis of simple porous models 3D-printed in aluminum, steel, ceramic, gypsum, and silica powders. The results demonstrate that powder materials can impart significant porosity as a byproduct of the printing process. The author provides implications of this porosity for repeatable flow and geomechanical experiments of porous media.

Ardila et al. investigate porous models 3D-printed with silica sand and furfuryl alcohol binder as well as interactions between these models and oil and water. Experimental results indicate mixed-wet characteristics of the 3D-printed models. The authors suggest optimizations to the 3D printing process to control the wettability of pore-network surfaces.

Gomez et al. investigate the suitability of sandstone analogs 3D-printed in silica sand to resemble the geomechanical behavior of Berea sandstone and their permeability evolution at each compression stage. While 3D-printed rock analogs are found to be more compressible 
and permeable than natural Berea sandstone, the authors propose a way to tune the $3 \mathrm{D}$ printing process to reduce disparities in mechanical and hydraulic behavior.

Perras and Vogler perform tensile and compressive geomechanical tests on models 3Dprinted in sand with furan and silicate binders (as well as in ceramic beads with silicate binder) using natural sandstones of varying strength and stiffness for experimental control. They demonstrate a promising capability of 3D-printed sand with furan binder to replicate the geomechanical behavior of natural sandstones.

Hodder and Nychka propose a new silane treatment method to change the wettability of polymer binder and silica sand to produce a hydrophilic surface. This method enables tunable wettability of 3D-printed sand for repeatable and direct geomechanical and flow experimentation under different environmental conditions.

Kong et al. present an optimal post-processing method of 3D-printed gypsum models that can facilitate replication of natural rocks with similar porosity. In addition, multifractal theory is used to understand the heterogeneity of pore-throat size distribution within 3D-printed samples and to evaluate possible flow pathways in the pore system of gypsum models.

\subsection{Nomenclature}

This section provides the most recent attempt to unify the terms used in studies of 3D-printed models from geological data because no uniform nomenclature exists in this field.

$3 D$-printed or printed both refer to a proxy, model, or object that was produced on a $3 \mathrm{D}$ printer.

$3 D$ printing resolution or $3 D$ printer's resolution the smallest element (e.g., "grain") or gap (e.g., "pore") that a 3D printer can produce.

$3 D$ printing a process of creating physical models layer-by-layer using a base material or feedstock (e.g., powder, plastic) and, if necessary, a support material (e.g., wax).

Analog rocks or reservoir analogs or artificial rocks models or proxies 3D-printed in one of the materials that resemble textural, geometrical, transport, or geomechanical properties of (reservoir) rocks.

Base or build material a bulk material used during 3D printing process to manufacture a physical model (e.g., plastics, powder, and resin).

Binder a liquid adhesive compound that is used in powder-based 3D printing to bond powder grains on each layer of the model.

Binder-jet printing a 3D printing technology where a binder is selectively deposited onto a powder bed, bonding otherwise loose grains to form a solid model one layer at a time. Powders may include gypsum, metals, ceramics, silica, etc.

Binder saturation the pore volume in a predefined total area that is filled with any binder.

CT data computed tomography data. The 3D penetrative volumetric image of a 3D model.

Computational (numerical) analysis a method that employs digital models of rocks to calculate properties and simulate processes. These numerical simulations are often calibrated using tangible samples (natural or fabricated) with experimental analysis.

Experimental (laboratory) analysis a method that employs destructive and nondestructive techniques to measure physical, chemical, mechanical, transport, electrical, or other properties of rocks or physical objects (like 3D-printed models).

Fracture network a model of a porous medium that contains fractures arranged in a 3D geometrical volume with specified sizes, connectivity, and shapes.

Infiltrant a liquid adhesive used in post-processing for models 3D-printed from powders for strengthening and sealing remaining pores between grains. 
Micromodel or microfluidic model transparent pore network that contains one or more interconnected channels in two or " 2.5 " dimensions. Such models enable the optical visualization of the fluid flow on very small length scales ( $<100$ microns).

Multijet modeling or polyjet printing a 3D printing technology where droplets of liquid polymer are instantly cured with UV light to build a model, layer-by-layer. Depending on the complexity of porous models, pores can be filled in with support material to hold the internal structure.

Numerical (digital) model cellular model of pores and voids that represents a porous 3D volume and exists within a computer. These models can be:

- Rock Numerical Models derived from CT data of a natural rock or sediment pack;

- Simple Numerical Models made by using digital design tools like CAD software. Simple proxies can be made subtractively (e.g., excising pores and pore throats from a "primitive" 3D shape like a cylinder) or additively (e.g., building a pore network from smaller parts leaving void spaces in between);

- Stochastic Numerical Models made by stochastically populating a 3D volume with matter and void based on idealized algorithms or training data from natural samples.

Particle Image Velocimetry a nondestructive optical measurement technique that allows tracking particle velocity during laboratory fluid transport experiments.

Pore network a model of a pore system that contains pore bodies and pore throats arranged in a 3D geometrical volume with specified sizes, connectivity, and shapes.

Post-processing a set of procedures intended to improve the quality and accuracy of a 3D-printed model and can involve removal of support material and excessive base material from internal and external surfaces.

Powder a type of 3D printing material used as the base material to create physical models in binder-jet printing (e.g., metals, ceramics, silica, and clay).

Proxy a 3D-printed example of a numerical model. It also refers to 3D-printed model or object. These can be:

- Rock Proxy proxy 3D-printed from a Rock Numerical Model;

- Simple Proxy proxy 3D-printed from a Simple Numerical Model;

- Stochastic Proxy proxy 3D-printed from a Stochastic Numerical Model.

Proxy accuracy a measure of how accurate a 3D printer can replicate geometry and sizes of pores and grains of a digital model in a proxy.

Stereolithography a 3D printing technology where the physical model is manufactured in a tank with liquid resin by a laser or optical light curing the liquid build material.

Support material a material used to hold the internal structure of a 3D-printed model. For example, wax is a common support material in multijet printing, which is removed from pores after 3D printing. Gypsum can be a support material itself when the pores of powder models are filled with dry gypsum after $3 \mathrm{D}$ printing.

Publisher's Note Springer Nature remains neutral with regard to jurisdictional claims in published maps and institutional affiliations. 